\title{
Distinct Urinary Metabolic Profile in Rheumatoid Arthritis Patients: A Possible Link between Diet and Arthritis Phenotype
}

\author{
Jung Hee Koh ${ }^{1, *}$, Yune-Jung Park ${ }^{2, *}$, Saseong Lee ${ }^{3}$, Young-Shick Hong ${ }^{4}$, Kwan Soo Hong ${ }^{5}$, Seung-Ah Yoo ${ }^{3}$, \\ Chul-Soo $\mathrm{Cho}^{2,3}$, Wan-Uk Kim ${ }^{2,3}$ \\ ${ }^{1}$ Division of Rheumatology, Department of Internal Medicine, Pusan National University Hospital, Busan, ${ }^{2}$ Division of Rheumatology, \\ Department of Internal Medicine, College of Medicine, The Catholic University of Korea, ${ }^{3}$ Center for Integrative Rheumatoid Transcriptomics and \\ Dynamics, The Catholic University of Korea, Seoul, ${ }^{4}$ Division of Food and Nutrition, Chonnam National University, Gwangju, ${ }^{5}$ Bioimaging \\ Research Team, Korea Basic Science Institute, Cheongju, Korea
}

Objective. We undertook this study to investigate the discriminant metabolites in urine from patients with established rheumatoid arthritis (RA), systemic lupus erythematosus (SLE), and from healthy individuals. Methods. Urine samples were collected from 148 RA patients, 41 SLE patients and 104 healthy participants. The urinary metabolomic profiles were assessed using ${ }^{1} \mathrm{H}$ nuclear magnetic resonance spectroscopy. The relationships between discriminant metabolites and clinical variables were assessed. Collagen-induced arthritis was induced in mice to determine if a choline-rich diet reduces arthritis progression. Results. The urinary metabolic fingerprint of patients with established RA differs from that of healthy controls and SLE patients. Markers of altered gut microbiota (trimethylamine-N-oxide, TMAO), and oxidative stress (dimethylamine) were upregulated in patients with RA. In contrast, markers of mitochondrial dysfunction (citrate and succinate) and metabolic waste products (p-cresol sulfate, $p$-CS) were downregulated in patients with RA. TMAO and dimethylamine were negatively associated with serum inflammatory markers in RA patients. In particular, patients with lower p-CS levels exhibited a more rapid radiographic progression over two years than did those with higher p-CS levels. The in vivo functional study demonstrated that mice fed with $1 \%$ choline, a source of TMAO experienced a less severe form of collagen-induced arthritis than did those fed a control diet. Conclusion. Patients with RA showed a distinct urinary metabolomics pattern. Urinary metabolites can reflect a pattern indicative of inflammation and accelerated radiographic progression of RA. A choline-rich diet reduces experimentally-induced arthritis. This finding suggests that the interaction between diet and the intestinal microbiota contributes to the RA phenotype. (J Rheum Dis 2019;26:46-56)

Key Words. Rheumatoid arthritis, Urine, Metabolomics, Trimethylamine-N-oxide, Diet

\section{INTRODUCTION}

Rheumatoid arthritis (RA) is a systemic inflammatory disease characterized by symmetric, peripheral polyarthritis. Pro-inflammatory cytokines play a key role in the pathogenesis of synovitis and systemic inflammation [1]. These cytokines regulate cachexia via synthesis of acute phase reactants and changes to metabolism that provide substrates and energy [2]. Stimulated immune cells consume more energy than do inactive immune cells [3].

The metabolic changes in RA have been described based on serum, urine and synovial fluid. The first distinct metabolite that was recognized in RA is tryptophan in the urine [4]. Soon after, low serum histidine levels were observed in RA in association with an elevated C-reactive protein [5]. More recently, one group was able to dis-

Received : September 11, 2018, Revised : October 30, 2018, Accepted : November 4, 2016

Corresponding to : Wan-Uk Kim (ib)http://orcid.org/0000-0001-8224-8496

Division of Rheumatology, Department of Internal Medicine, Seoul St. Mary's Hospital, College of Medicine, The Catholic University of Korea, 222 Banpo-daero, Seocho-gu, Seoul 06591, Korea. E-mail : wan725@catholic.ac.kr

*These authors contributed equally to this work.

Copyright (c) 2019 by The Korean College of Rheumatology. All rights reserved.

This is a Open Access article, which permits unrestricted non-commerical use, distribution, and reproduction in any medium, provided the original work is properly cited. 
tinguish RA patients who have a good response to anti-tumor necrosis factor- $\alpha$ (TNF- $\alpha$ ) therapy from those who do not based on their urine metabolic profiles [6]. Analysis of the metabolites in RA has allowed for improved understanding of the disease's nature, of the relationship between the metabolic status and disease activity, and of specific drug response on the metabolic profile and disease subtype differentiation. Metabolomics is among the new family of the "-omics". It refers to the systematic and comprehensive assessment of endogenous metabolites [7]. Metabolomics is a non-targeted approach used to identify biomarkers and provide patient profiling, in line with systems biology.

The etiology of RA is not fully understood. In general, disruptive pathological processes can result in altered metabolite profiles long before overt arthritic symptoms appear in RA. In addition, circulating pro-inflammatory cytokines, acute phase reactants and immune complexes also affect metabolism. Therefore, it is not surprising that there is increasing interest in using altered metabolites as biomarkers of disease prediction, diagnosis, activity and response to therapy.

Previously, our group analyzed the urinary proteome profile and demonstrated that urinary soluble CD14 has diagnostic value and high predictive power for disease activity [8]. In order to investigate the potential of metabolic fingerprinting in RA, we applied a nuclear magnetic resonance (NMR)-based metabolomics approach to the analysis of RA patients. In addition, the metabolites represent the intermediate products of physiological processes that are influenced by the mechanisms of disease, environment, microbiota, and the digestion and biotransformation of foods and their constituents. We further studied the in vivo role of metabolites in the animal arthritis model and human clinical data.

\section{MATERIALS AND METHODS}

\section{Study population}

We collected random urine samples from 148 RA patients, 41 systemic lupus erythematosus (SLE) patients and 104 healthy volunteers. The study protocol was approved by the Institutional Review Board of the Catholic Medical Center (KC16SISI0632). Informed consent was obtained from all participants.

We recorded the following clinical data at the time of urine sampling: laboratory data; Disease Activity Score in 28 joints using the erythrocyte sedimentation rate (DAS
28-ESR); and prescribed medications. The van der Heijde-modified Sharp Score (HSS) [9] was measured at baseline and at the two-year follow-up period. The radiographic progression ( $\triangle \mathrm{HSS}$ ) was calculated by the difference in the HSS between the baseline and two-year measurements. A clinically significant change $(\triangle \mathrm{HSS})$ was defined as $>5[10]$.

\section{Metabolomic analysis}

Urine metabolomics profiles were assessed using ${ }^{1} \mathrm{H}$ NMR spectroscopy. The detailed methods are available in the supplementary materials.

\section{NMR data preprocessing and statistical analysis}

The NMR spectra were imported into MATLAB (R2010b; Mathworks, Inc., 2010, Natick, MA, USA), with no binning or spectral segments, using in-house software. We then performed probabilistic quotient normalization of the spectra using the mean spectrum to estimate the most probabilistic quotient after total integral normalization following removal of the region corresponding to residual water ( $\delta 4.57$ to 5.20 ). Total integral normalization was performed to avoid dilution effects of the urine samples and effects of metabolites in massive amount on changes to the overall concentration of the urine samples [11].

The resultant data were analyzed using multivariate statistical models, such as principal component analysis (PCA), partial least-squares discriminant analysis (PLS-DA), and orthogonal projections to the latent structures discriminant analysis (OPLS-DA) [12]. In addition, the OPLS loading coefficient or the OPLS loading plots in the OPLS-DA models between two classes were back-scaled to improve the interpretability and identify discriminant urinary metabolites, as described by Cloarec et al. $[13,14]$ In order to prevent over-fitting the spectra data in the OPLS-DA models, the 7-fold cross validation method was used. We also calculated the cross-validation parameter $Q^{2}$.

\section{Induction of collagen-induced arthritis in mice}

We used the collagen-induced arthritis (CIA) model to study the potential contribution of a continuous choline-rich diet, a source of trimethylamine- $\mathrm{N}$-oxide (TMAO), to arthritis severity. The conventional mice (DBA/1) were obtained from Japan SLC Inc. (Shizuoka, Japan) at 4 weeks of age. The mice were maintained in specific pathogen-free conditions. The animal study was 
approved by the Institutional Animal Care and Use Committee of the Catholic University of Korea (CMC IACUC 2016-0202-03). Male DBA1 mice were placed on either a standard chow diet (Teklad 2018) or $1 \%$ choline supplemented diet (Teklad TD130328) at least 4 weeks before inducing CIA. The DBA/1 mice were immunized with bovine type II collagen (CII; Chondrex Inc., Redmond, WA, USA) emulsified in Complete Freund's adjuvant (CFA; Chondrex Inc.). Fourteen days after the primary immunizations, these animals received a booster immunization with CII emulsified in Incomplete Freund's adjuvant (IFA; Chondrex Inc.) [15]. Starting 21 days after the primary immunization, the severity of the arthritis was assessed by two independent observers, three times per week. The severity of joint inflammation was evaluated using the arthritis index and paw swelling [16].

For histological assessment of the arthritic joints, mice were euthanized in an isoflurane chamber. Death was confirmed by cervical dislocation. The joint tissues were fixed in $4 \%(\mathrm{v} / \mathrm{v})$ paraformaldehyde, decalcified in a $10 \%$ Formic acid, embedded in paraffin, and cut into sections. The sections $(7 \mu \mathrm{m})$ were stained with hematoxylin and eosin. The extent of inflammation, bone destruction and synovial hyperplasia were scored as described previously $[16,17]$.

\section{Isolation and polarization of bone-marrow-de- rived macrophages (BMDM)}

Mice either received a $1 \%$ choline diet or control diet for at least 4 weeks in order to test the plasticity of macrophages during development. Bone marrow cells were extracted from the femur and differentiated in RPMI 1640 containing $10 \% \mathrm{FBS}$, and supplemented with $10 \mathrm{ng} / \mathrm{mL}$ monocyte-colony stimulating factor (M-CSF) at $37^{\circ} \mathrm{C}$ in a $5 \% \mathrm{CO}_{2}$ atmosphere for 6 days. The BMDM were counted and replated for polarized activation: $5 \times 10^{5} \mathrm{BMDMs}$ per milliliter were used for the in vitro experiments. We next sought to induce polarization of the BMDM into M1 or M2 macrophages. To create M1 macrophages, the cells were cultured with M-CSF $(10 \mathrm{ng} / \mathrm{mL})$ for 24 hours and then stimulated with interferon- $\gamma(50 \mathrm{ng} / \mathrm{mL})$ and lipopolysaccharide $(100 \mathrm{ng} / \mathrm{mL})$ for 24 hours. In contrast, for M2 macrophages, cells were cultured with interleukin (IL)-4 (10 ng/mL) and IL-13 (10 ng/mL) for 24 hours.

\section{Real-time polymerase chain reaction (PCR)}

Total RNA was isolated using the RNeasy Mini kit
(QIAGEN, Hilden, Germany). cDNA was prepared using $100 \mathrm{ng} / \mathrm{mL}$ total RNA by reverse-transcription using reverse transcriptase (Takara, Shiga, Japan) according to the manufacturer's instructions. Real-time quantitative PCR was performed using a CFX96 machine (Bio-Rad, Hercules, CA, USA) using SYBR Green PCR Master Mix (Bio-Rad) and primers for IL-6, arginase 1, and inducible nitric oxide synthase (iNOS). Glyceraldehyde 3-phosphate dehydrogenase (GAPDH) was used as the internal control for PCR amplification. The primers that were used are listed in Supplementary Table 1. Transcript levels were calculated relative to those of the controls, and are expressed as $-\triangle \Delta \mathrm{Ct}$.

\section{Enzyme-linked immunosorbent assay (ELISA) for IL-6}

The cytokine concentrations in the cell culture supernatants were measured using ELISA Duoset (R\&D Systems, Minneapolis, MN, USA) kits for mouse IL-6 according to the manufacturer's instructions.

\section{Statistical analysis}

Statistical analyses were performed using SAS 9.4 (SAS Institute Inc., Cary, NC, USA). The Shapiro-Wilk test was used to test the continuous outcome variables for their normality of distribution. Comparisons between groups were made using the Student's t-test or Mann-Whitney U-test, as appropriate. The arthritis score from the two dietary groups were compared using repeated measures ANOVA with Greenhouse-Geisser correction. This analysis allowed us to assess the development of CIA over time. The categorical data were compared using $\chi^{2}$ test or Fisher's exact test. A p-value $<0.05$ was considered statistically significant. Spearman correlations were used to make comparisons between selected urinary metabolites and clinical indices. Multivariate logistic regression was performed to determine the effects of urinary metabolites, cytokines and acute phase reactants on radiographic progression in patients with RA.

\section{RESULTS}

\section{Comparing the urinary metabolomics profiles in patients with RA and SLE to those of healthy controls}

The whole ${ }^{1} \mathrm{H}$ NMR spectrum of 293 urine samples, consisting of 23 metabolites, was applied to multivariate statistical analysis (such as PCA and OPLS-DA). This techni- 
que was used to identify outliers, and to determine whether SLE and RA patients had unique urinary profiles to those of healthy volunteers (Figure 1A $\sim$ E). Outliers with markedly increased levels of urinary TMAO and di- methylamine (Supplementary Figure 1) were removed from further analysis. Patients with SLE and RA were differentiated from healthy volunteers using the OPLS-DA score plot (Figure 1E). The OPLS-DA model was vali-

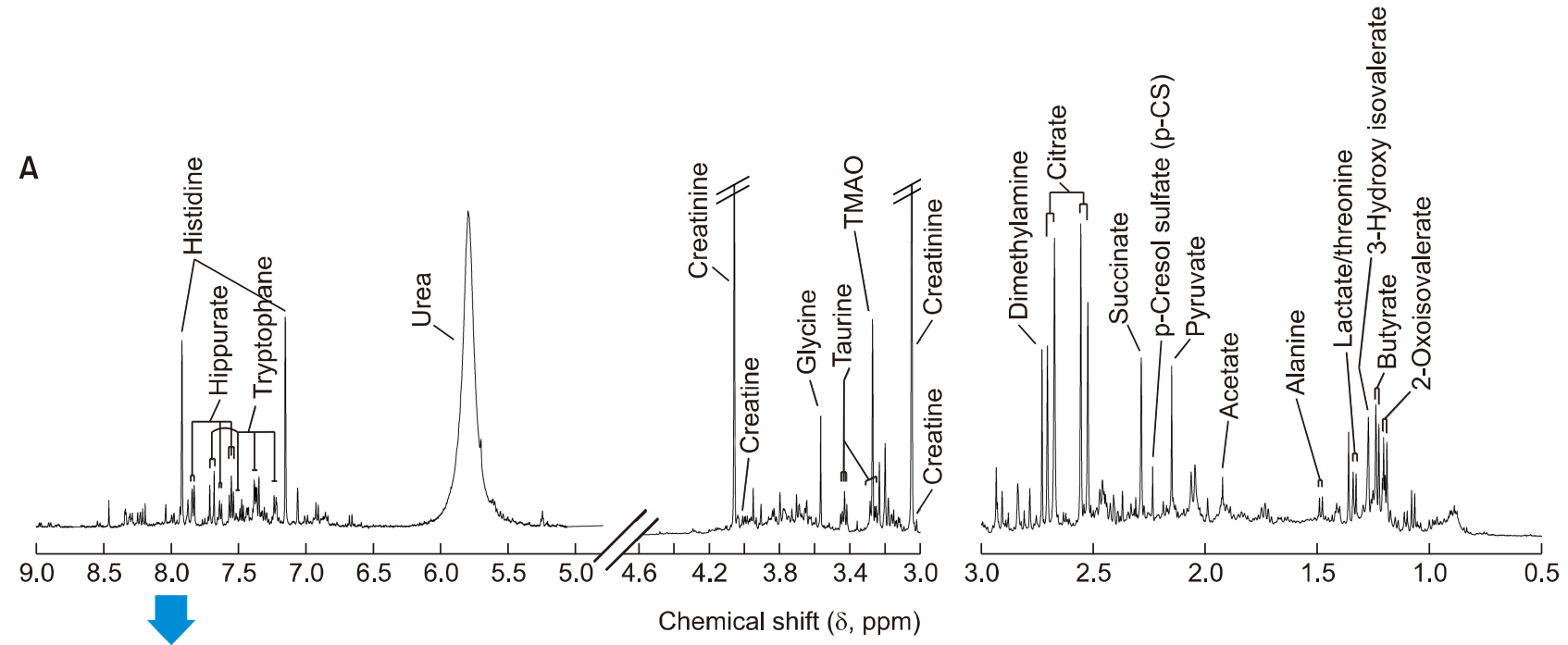

B
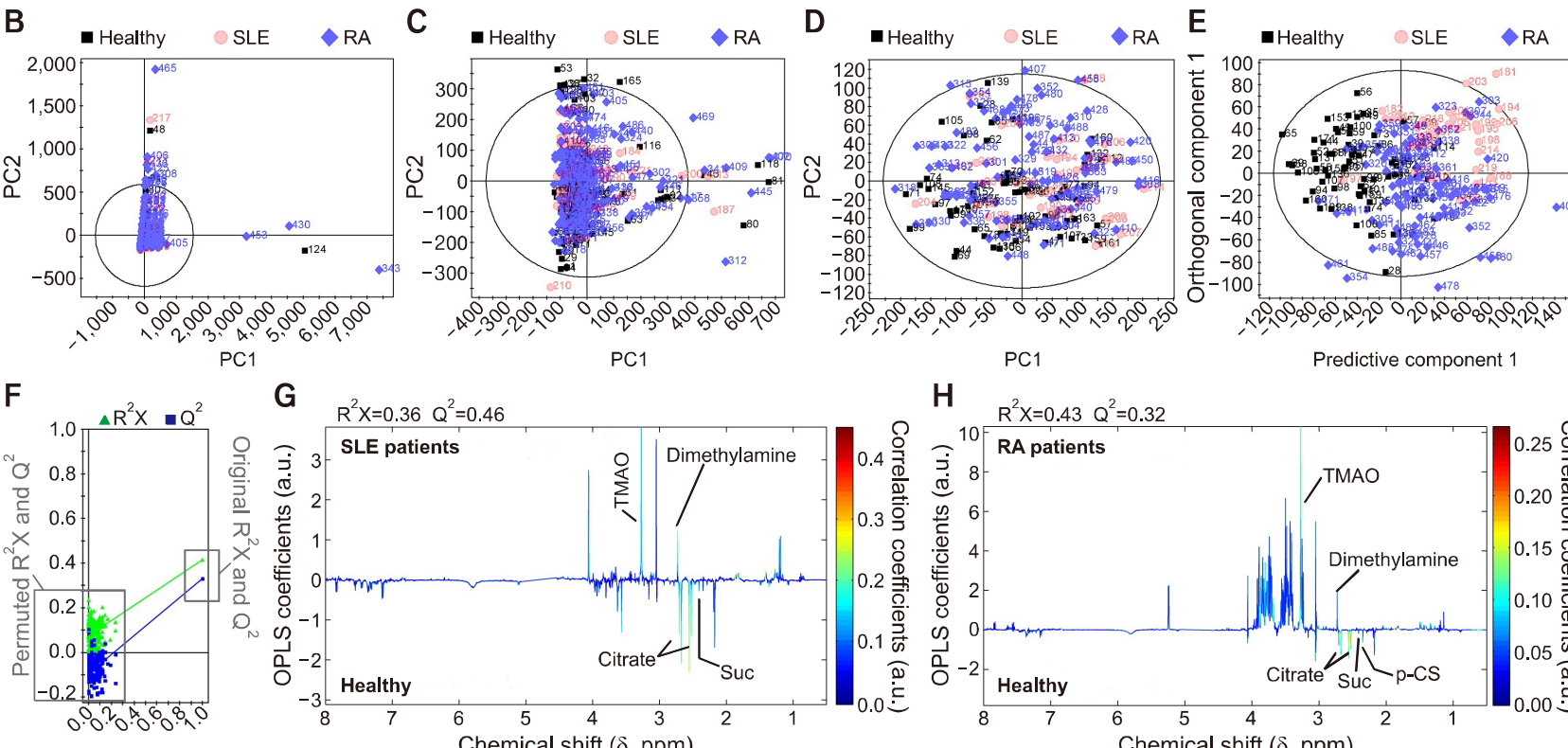

G

$\mathrm{H}$
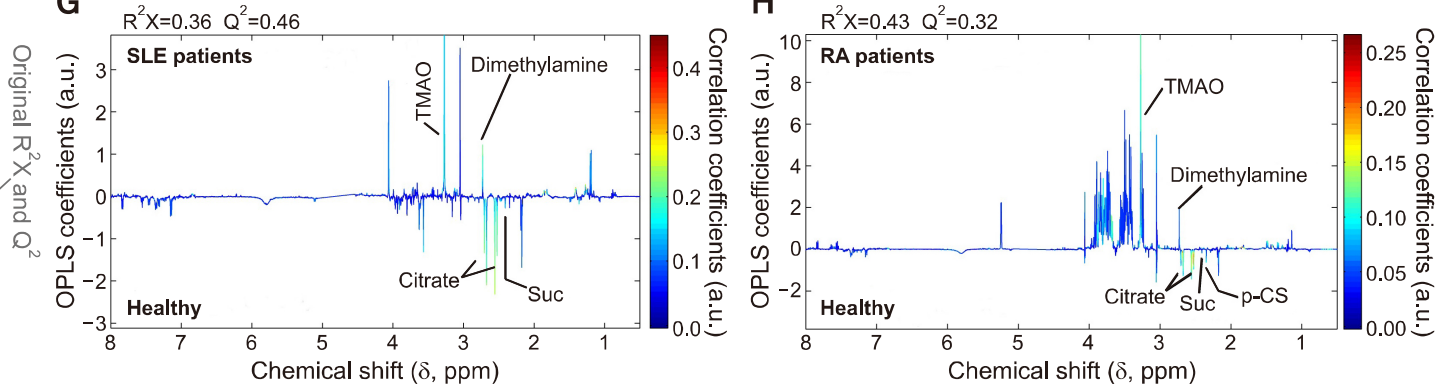

Figure 1. Representative ${ }^{1} \mathrm{H}$ NMR spectrum of human urine (A). Multivariate statistical analysis including PCA (B D) and OPLS-DA models ( $E$ and $F$, the permutation test for validation of the OPLS-DA models in the panel F) derived from ${ }^{1} \mathrm{H} N M R$ urinary spectra; a pairwise comparison of urinary metabolites between healthy volunteers and SLE patients (G); and between healthy volunteers and RA patients $(\mathrm{H})$ in OPLS-loading plot for effective findings of urinary metabolites that differ between two classes. The OPLS-DA models in the panels $(\mathrm{E})$ through $(\mathrm{H})$ were displayed following removal of outliers observed in the PCA model analysis. The urinary TMAO was mainly responsible for outlying human urines including healthy volunteers and arthritis patients in multivariate statistical analysis. Panel (F) reflects validation of the OPLS-DA model in panel (E) through 200 times permutation test, in which the original $Q^{2}$ value was higher than the $Q^{2}$ values permuted. The upper section in the OPLS-loading plots in the panels $(G$ and $H$ ) represent the increased urinary metabolites in SLE and RA patients compared to those of healthy volunteers. NMR: nuclear magnetic resonance, PCA: principal component analysis, OPLS-DA: orthogonal projections to the latent structures discriminant analysis, SLE: systemic lupus erythematosus, RA: rheumatoid arthritis, TMAO: trimethylamine-N-oxide, a.u.: arbitrary unit. 
Jung Hee Koh et al.

dated using the permutation test (Figure $1 \mathrm{~F} \sim \mathrm{H}$ ). Urinary TMAO and dimethylamine levels were also increased in both SLE and RA patients, compared to those of healthy volunteers (Figure $2 \mathrm{~A} \sim \mathrm{D}$ ) based on the observed outliers in the healthy volunteers (Supplementary Figure 1). However, the levels of urinary citrate and succi-
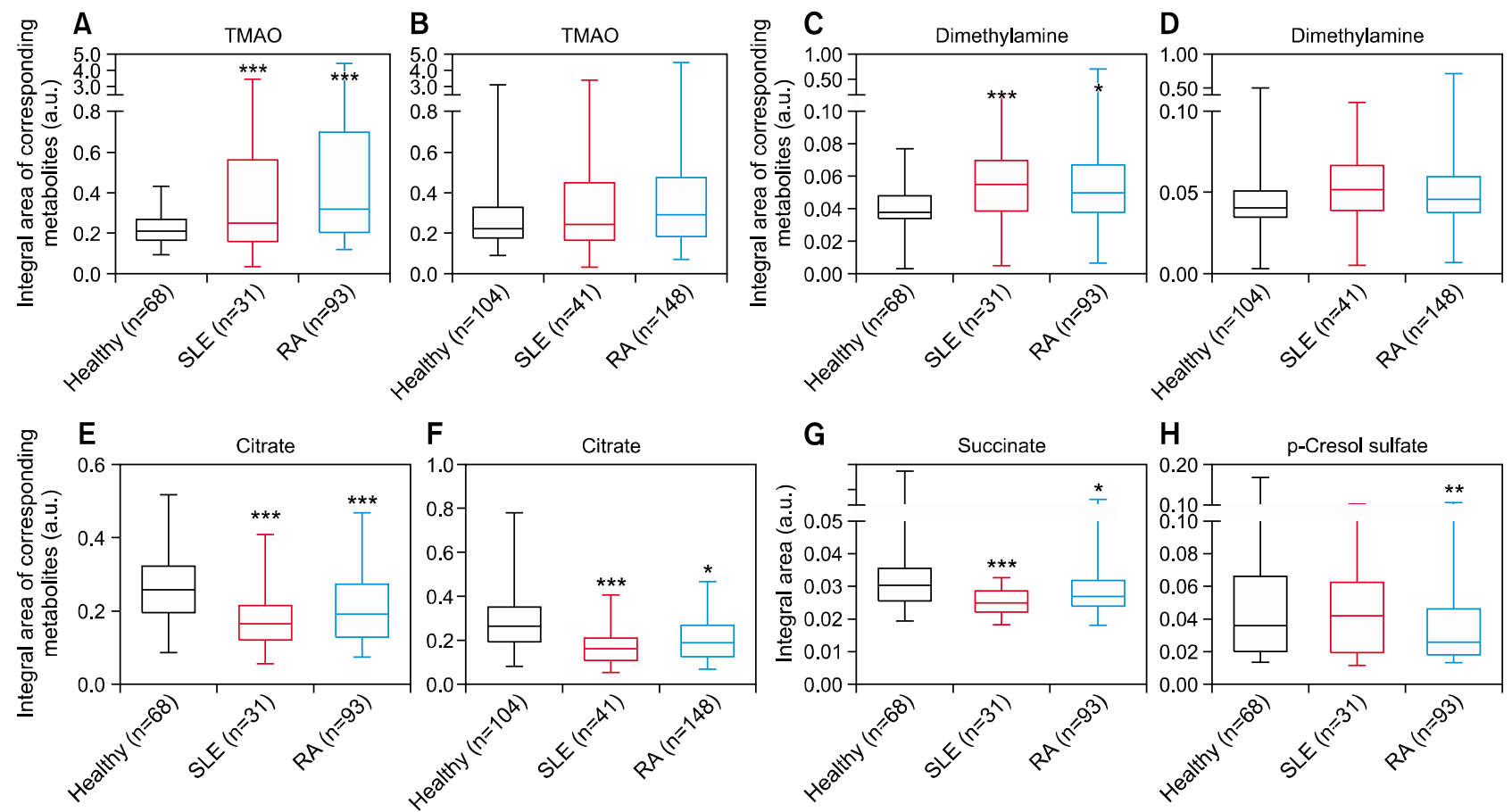

Figure 2. Comparison of individual metabolites between healthy volunteers, patients with rheumatoid arthritis (RA), and systemic lupus erythematosus (SLE). Data in panels (A, C, E, G, and H) were displayed following removal of outliers observed in the principal component analysis (PCA) model analysis. All samples, including the outliers, removed from the PCA models were included in the statistical analysis in panels (B, D, and F). TMAO: trimethylamine-N-oxide, a.u.: arbitrary unit. ${ }^{*} \mathrm{p}<0.05,{ }^{* *} \mathrm{p}<0.01,{ }^{* * *} \mathrm{p}<0.001 \mathrm{vs}$. healthy volunteers.

Table 1. Baseline patient characteristics by group

\begin{tabular}{|c|c|c|c|c|c|c|}
\hline Variable & $\begin{array}{l}\text { Healthy controls } \\
\qquad(\mathrm{n}=68)\end{array}$ & $\begin{array}{l}\text { Patients with } \\
\text { SLE }(n=31)\end{array}$ & $\begin{array}{l}\text { Patients with RA } \\
\qquad(\mathrm{n}=93)\end{array}$ & $\begin{array}{c}\text { p-value } \\
\text { (HC vs. SLE) }\end{array}$ & $\begin{array}{c}\text { p-value } \\
\text { (HC vs. RA) }\end{array}$ & $\begin{array}{c}p \text {-value } \\
\text { (SLE vs. RA) }\end{array}$ \\
\hline Age (yr) & $54(47 \sim 57)$ & $41(34 \sim 49)$ & $52(45 \sim 61)$ & $<0.001$ & 0.916 & $<0.001$ \\
\hline Female & $65(95.6)$ & $30(96.8)$ & $76(81.7)$ & $>0.999$ & 0.008 & 0.042 \\
\hline Hypertension & $16(23.5)$ & $5(16.1)$ & $24(25.8)$ & 0.496 & 0.445 & 0.957 \\
\hline Diabetes mellitus & $4(5.9)$ & $1(3.2)$ & $6(6.4)$ & 0.576 & 0.882 & 0.500 \\
\hline Symptom duration (yr) & NA & $4(2 \sim 7)$ & $5(2 \sim 10)$ & NA & NA & 0.354 \\
\hline RF positive* & NA & NA & $62(66.7)$ & NA & NA & NA \\
\hline ACPA positive* & NA & NA & $74(79.6)$ & NA & NA & NA \\
\hline ESR $(\mathrm{mm} / \mathrm{hr})$ & NA & NA & $28(19 \sim 47)$ & NA & NA & NA \\
\hline $\mathrm{CRP}(\mathrm{mg} / \mathrm{dL})$ & NA & NA & $0.3(0.1 \sim 1.6)$ & NA & NA & NA \\
\hline Prednisolone & NA & $22(70.9)$ & $79(84.9)$ & NA & NA & 0.561 \\
\hline NSAIDs & NA & $9(29.0)$ & $45(48.4)$ & NA & NA & 0.027 \\
\hline Methotrexate & NA & $5(16.1)$ & $54(58.1)$ & NA & NA & $<0.001$ \\
\hline Hydroxychloroquine & NA & $24(77.4)$ & $64(68.8)$ & NA & NA & 0.015 \\
\hline Anti-TNF- $\alpha$ & NA & $\mathrm{NA}$ & $11(11.8)$ & NA & NA & $\mathrm{NA}$ \\
\hline
\end{tabular}

Data are presented as medians (interquartile ranges) or numbers (percentages). SLE: systemic lupus erythematosus, RA: rheumatoid arthritis, HC: healthy controls, RF: rheumatoid factor, ACPA: anti-cyclic citrullinated peptide antibody, ESR: erythrocyte sedimentation rate, CRP: C-reactive protein, NSAIDs: non-steroidal anti-inflammatory drugs, TNF- $\alpha$ : tumor necrosis factor- $\alpha$, NA: not applicable. *Antibody positivity. Positive cut-off values were $\geq 15 \mathrm{IU} / \mathrm{mL}$ for RF and $>5 \mathrm{U} / \mathrm{mL}$ for ACPA, respectively. 
nate were further decreased in patients with SLE and RA (Figure $2 \mathrm{E} \sim \mathrm{G}$ ) compared to those values of healthy volunteers. Interestingly, significant decreases in urinary $\mathrm{p}$-cresol sulfate ( $\mathrm{p}-\mathrm{CS}$ ) levels (compared to those of healthy volunteers) were only observed in RA patients (Figure 2H).

Five urinary metabolites were significantly perturbed in the patients with SLE and RA. These changes are likely associated with biologically relevant pathways, such as markers of altered gut microbiota (TMAO), mitochondrial dysfunction (succinate and citrate; citric acid cycle intermediates), oxidative stress (dimethylamine, purine metabolites), and metabolic waste products (p-CS). The baseline characteristics of all included patients are given in Table 1.

\section{Associations of urinary metabolites with disease activity and radiographic RA progression}

We next examined the inter-relationships between urinary metabolites and markers of systemic inflammation and joint destruction. Three distinct sub-clusters of urinary metabolites were identified by correlation analysis, and are as follows: citrate and succinate (in sub-cluster 1); TMAO and dimethylamine (in sub-cluster 2); and p-CS (in sub-cluster 3) (Figure 3A). Among the distinct urinary metabolites, TMAO and dimethylamine (in sub-cluster 2) were positively correlated with hemoglobin levels and negatively correlated with systemic markers of inflammation (Figure 3B). These results suggest that there are inter-relationships between the systemic and urinary metabolome profiles.

We compared urine metabolite profiles according to the radiographic progression to identify any metabolites associated with radiographic RA severity. Five metabolites could be differentiated according to radiographic severity. However, p-CS was the only metabolite that reached statistical significance following multiple testing corrections (Figure 3C and 3D). RA patients with higher quartiles of urinary p-CS demonstrated less radiographic progression than did those in lower quartiles (Figure 3D).

\section{Dietary choline reduces inflammatory arthritis}

The TMAO and inflammatory markers were negatively correlated. This result suggests that gut microbiota and specific dietary nutrients that enhance TMAO generation are associated with arthritis severity. To explore this possibility, we investigated whether continuous $1 \%$ choline diet affects arthritis severity in the mouse CIA model.
Mice were either placed on a high choline diet (1\% total choline) or normal chow diet ( $0.08 \%$ total choline). Mice that received the $1 \%$ choline diet developed less severe arthritis than did those that ate the normal chow diet (Figure 4A). Histologic joint examination also demonstrated less joint destruction in mice with $1 \%$ choline diet compared to that in mice that ate a normal chow diet (Figure 4B).

We hypothesized that the metabolites produced by a continuous choline-rich diet influence the immune response in RA. Macrophages were considered a target of TMAO [18]. We investigated whether a choline-rich diet is associated with the activation of the following two different macrophage stages: M1 (classical, inflammatory) and M2 (alternative, anti-inflammatory, tissue repair) $[19,20]$. To examine this association, we isolated BMDMs from the mice fed a $1 \%$ choline diet and normal chow diet for at least 4 weeks. The BMDMs were then polarized to M1 and M2 macrophages. We later compared the mRNA expression degrees of iNOS and Arginase 1. iNOS was more highly expressed in M1 macrophages from mice fed with a normal chow diet than it was in those fed with a $1 \%$ choline diet. In contrast, Arginase 1 was modestly more highly expressed in M2 macrophages from mice fed with a $1 \%$ choline diet than it was in those fed with a normal chow diet (Figure 4C and 4D). The IL-6 expression was also significantly increased in M1 macrophages from mice fed with a normal chow diet compared to those fed with the $1 \%$ choline diet (Figure $4 \mathrm{E}$ and $4 \mathrm{~F}$ ).

\section{DISCUSSION}

Our data demonstrate that the urinary metabolite fingerprint of patients with RA differs from those of healthy volunteers and patients with SLE. To the best of our knowledge, this study is the first to examine urine metabolomics in a large cohort of patients in which those with RA were compared to healthy individuals and patients with SLE. It is also the first to use an animal arthritis model to investigate the relationship between dietary choline and arthritis severity. TMAO and dimethylamine were upregulated in the urine samples from patients with RA. In contrast, citrate, succinate and p-CS were downregulated in RA patients. Our study also suggests that a high choline diet can modulate arthritis via macrophage plasticity.

Elevated TMAO promotes foam cell production by increasing the macrophage surface expression of pro-athe- 

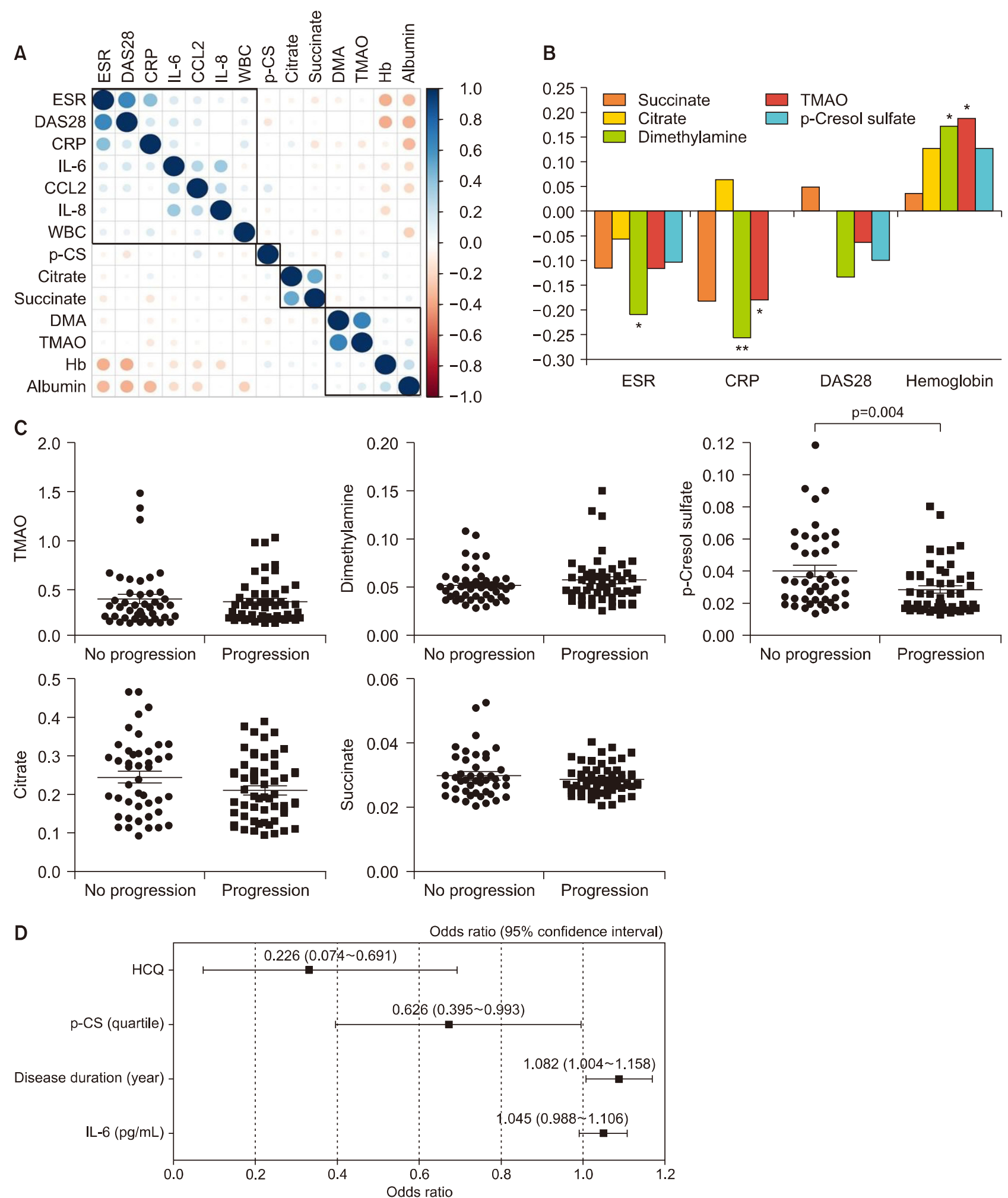

Figure 3. Associations between urinary metabolites, inflammation and radiographic progression in rheumatoid arthritis (RA) patients. (A) Cluster correlation analysis. The power of the correlation is represented by the color and size of the circles. (B) Correlation of urinary metabolite levels with clinical inflammatory variables measured in RA patients. (C) Comparison of urinary metabolite levels in patients with radiographic progression $(n=49)$ and without progression $(n=44)$. (D) Multivariate logistic regression analysis for predicting radiographic progression using conventional risk factors plus urinary metabolites. ESR: erythrocyte sedimentation rate, DAS28: Disease Activity Score 28, CRP: C-reactive protein, IL: interleukin, WBC: white blood cells, DMA: dimethylamine, TMAO: trimethylamine-N-oxide, Hb: hemoglobin, HCQ: hydroxychloroquine, p-CS: $\mathrm{p}$-cresol sulfate. ${ }^{*} \mathrm{p}<0.05$ and ${ }^{*} \mathrm{p}<0.01$. 
A

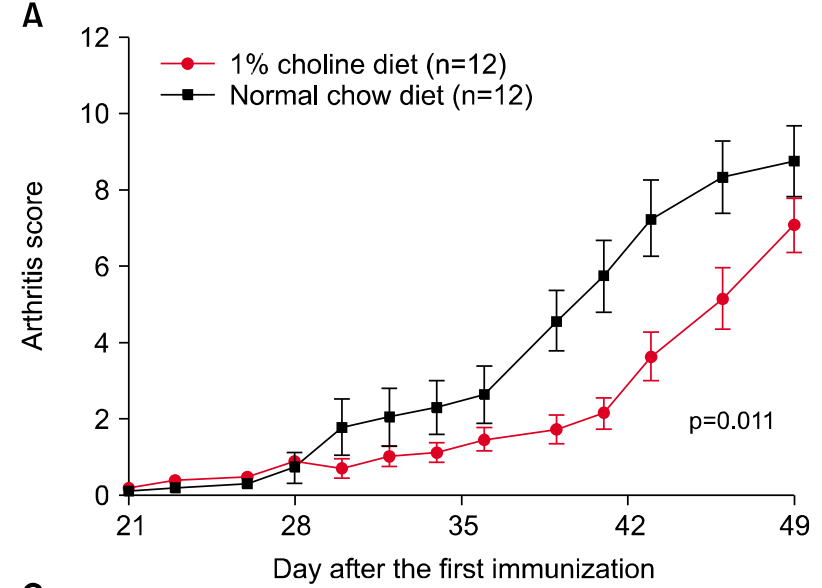

C

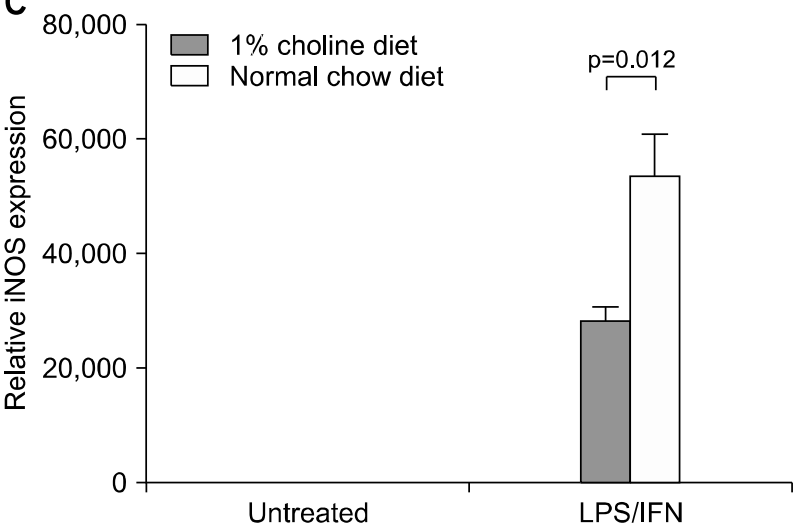

E

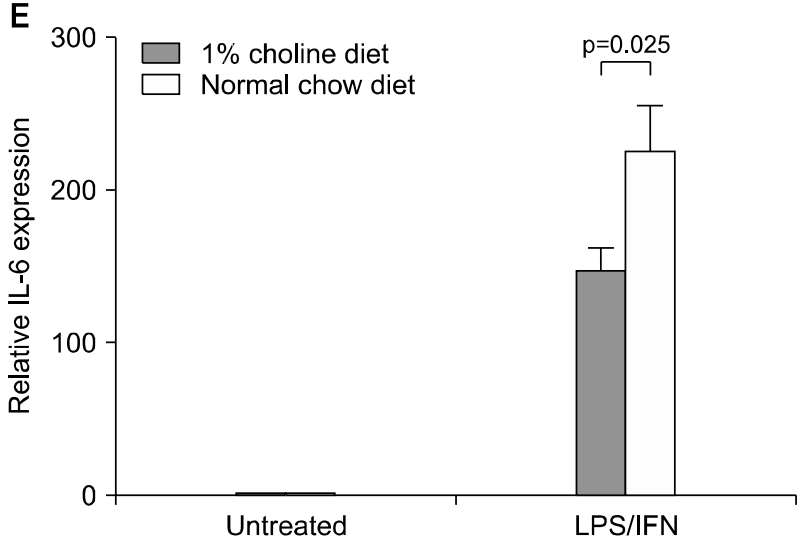

B
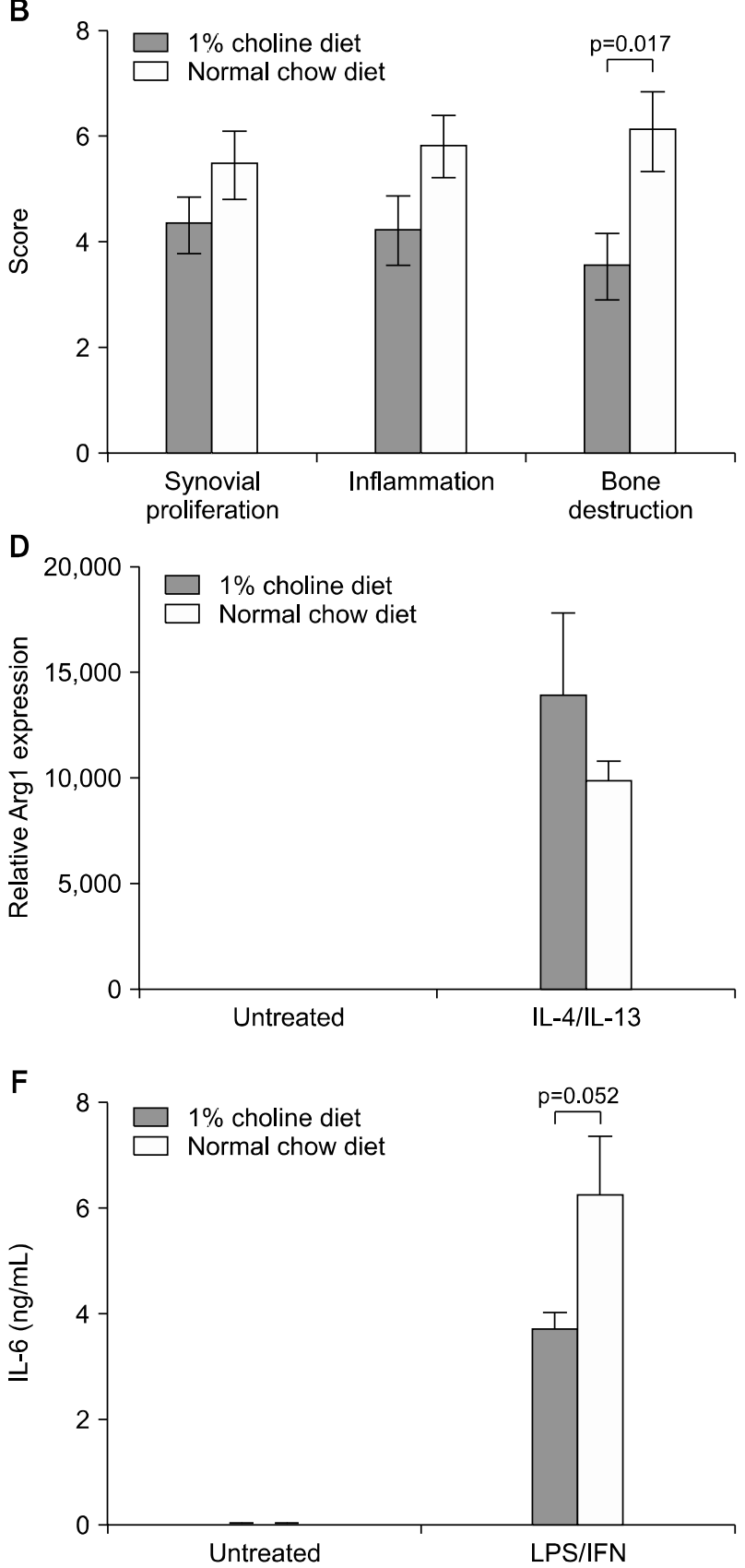

Figure 4. Dietary choline decreases the severity of collagen-induced arthritis in mice and inhibits macrophage activation. (A) Arthritis development was assessed by measuring the arthritis score. The $p$-value was calculated by repeated measures ANOVA using the Greenhouse-Geisser correction. (B) Quantification of histologic mouse arthritis score. (C to F) BMDMs were stimulated with interferon (IFN)- $\gamma(50 \mathrm{ng} / \mathrm{mL})$ and lipopolysaccharide (LPS) $(100 \mathrm{ng} / \mathrm{mL})$ or interleukin (IL)-4 (10 ng/mL) plus IL-13 (10 ng/mL) for 24 hours. mRNA was extracted from total cell lysates and analyzed by quantitative polymerase chain reaction (qPCR) for inducible nitric oxide synthase (iNOS) (C), Arginase 1 (Arg1, D), and IL-6 (E) expression. Supernatants were analyzed by enzyme-linked immunosorbent assay (ELISA) for IL-6 (F). The data are represented by means \pm standard errors of the mean of 10 independent experiments.

rogenic scavenger receptors [18]. It also induces multiple inflammatory proteins in human endothelial cells in vitro, including IL-6, E-selectin, and inter cellular adhesion molecule 1 [21], suggesting a pro-inflammatory role of
TMAO. Presently, we identified TMAO to be a metabolite that acts as a strong discriminator of RA from healthy subjects in the urine. However, the urinary TMAO level was negatively correlated with inflammatory markers in 
patients with RA, which is in sharp contrast with earlier reports $[18,21]$. Trimethylamine (TMA) is derived from a diet rich in phosphatidylcholine (major sources including eggs and red meat) through intestinal microbial processing; TMA is subsequently oxidized to TMAO by enzymes in the liver [22]. It has been demonstrated that the oral and gut microbiomes are perturbed in RA patients, which is partly normalized after treatment [23,24]. In addition, untreated RA patients carry high levels of Prevotella copri in stool [24] and the high abundance of gut Prevotella correlates with TMAO levels [25]. Interestingly, the composition of gut microbiome can be changed by non-antibiotic drugs, including disease-modifying anti-rheumatic drugs $[23,26]$. We speculate that dysbiosis may be linked to elevated levels of TMAO in RA patients, and such elevation can be reduced by treatment with anti-rheumatic drugs that normalize gut microbiomes as well as serum inflammatory markers.

p-CS originates from the bacterial metabolism of tyrosine in the intestine [27]. An elevated p-CS level serves as an inflammatory marker in patients with chronic kidney disease. It is also associated with increased arterial stiffness, inflammation, and oxidative stress [28]. Although the pathogenic role of p-CS in RA is not clear, its low level in the urine was a strong discriminator of RA patients from healthy volunteers and SLE patients. Urinary metabolites such as p-CS can also reflect accelerated radiographic progression of RA.

Patients with RA and SLE have been found to have considerably lower levels of succinate and citrate, substrates in the citric acid cycle, compared to those of healthy volunteers. The abundance of citrate in the synovial fluid was also lower in patients with RA than it was in those with non-RA [29]. These findings are consistent with a recent report that fibroblast-like synoviocytes display an altered pattern of glucose metabolism and attenuated mitochondrial respiration [30]. Activated T cells convert to aerobic glycolysis and low flux of the citric acid cycle [31]. These distinct metabolic signatures of RA and SLE may be used to diagnose and treat these diseases. However, additional studies are needed to confirm the assumption that these urinary metabolites are in steady state concentrations in an individual longitudinally.

In the present study, a long-term high-choline diet attenuated arthritis severity in mice with CIA. Moreover, the onset of arthritis was delayed in mice fed with high-choline diet (data not shown). Interestingly, BMDM from mice fed with a $1 \%$ choline diet demonstrated less M1 po- larization (as measured by iNOS upregulation) than did those of mice fed a normal chow diet. Together, the production of IL-6, a proinflammatory cytokine, was also reduced in M1 macrophages from mice fed with a $1 \%$ choline diet compared to that of mice fed a normal chow diet. Macrophages are abundant in the synovial tissue from RA patients and are associated with bone destruction [32]. Therefore, we suspected that a choline rich diet would affect synovial macrophages and attenuate inflammation in RA. Our results suggest that there is an additional nutritional contribution to the severity of RA that involves choline metabolism, intestinal microbiota and regulation of murine macrophage $\mathrm{M} 1$ and $\mathrm{M} 2$ polarization.

This study has several potential limitations. First, we were unable to exclude the possibility of the false positive results of urinary metabolites as a discriminator for RA from healthy controls by removing outliers. Second, intestinal microbiota are affected by habitual long-term diet and medications $[26,33]$. Therefore, a choline-rich diet could alter microbial composition, and produce different metabolites depending on the altered microbiota. Therefore, dietary challenges in a germ-free animal model with microbiome analyses will be necessary to elucidate the specific metabolites or microbiota that are associated with arthritis. It is also necessary to study the mechanism by which a high-choline diet affects M1 and M2 macrophage polarization. Third, various foods, such as red meat, eggs and fish, are important dietary sources of TMAO and its precursors (carnitine and choline) [34]. We did not investigate the dietary patterns of the participants; therefore, we were unable to determine which dietary components influenced the levels of urinary TMAO. Further studies of patients' dietary patterns are necessary to ultimately determine whether diet itself can be used to alleviate RA symptoms.

\section{CONCLUSION}

Patients with RA had distinct urinary metabolomics patterns compared to those of healthy individuals and patients with SLE. Urinary metabolites can serve as indicators of inflammation or accelerated radiographic progression of RA. A choline-rich diet reduces experimentally-induced arthritis, which suggests that there is an interaction between diet and intestinal microbiota that contributes to the RA phenotype. 


\section{ACKNOWLEDGMENTS}

We would like to thank all of the members of the Institute of Bone and Joint Diseases at the Catholic University of Korea. This research was supported by grants of grants from the National Research Foundation of Korea (NRF) funded by the Ministry of Science and ICT (2015R1A3A2032927 and 2018R1D1A1B07045491).

\section{CONFLICT OF INTEREST}

No potential conflict of interest relevant to this article was reported.

\section{SUPPLEMENTARY DATA}

Supplementary data can be found with this article online at https://doi.org/10.4078/jrd.2019.26.1.46.

\section{REFERENCES}

1. McInnes IB, Schett G. The pathogenesis of rheumatoid arthritis. N Engl J Med 2011;365:2205-19.

2. Kotler DP. Cachexia. Ann Intern Med 2000;133:622-34.

3. Kuhnke A, Burmester GR, Krauss S, Buttgereit F. Bioenergetics of immune cells to assess rheumatic disease activity and efficacy of glucocorticoid treatment. Ann Rheum Dis 2003; 62:133-9.

4. Bett IM. Metabolism of tryptophan in rheumatoid arthritis. Ann Rheum Dis 1962;21:63-9.

5. Sitton NG, Dixon JS, Bird HA, Wright V. Serum biochemistry in rheumatoid arthritis, seronegative arthropathies, osteoarthritis, SLE and normal subjects. $\mathrm{Br} \mathrm{J}$ Rheumatol 1987;26:131-5.

6. Kapoor SR, Filer A, Fitzpatrick MA, Fisher BA, Taylor PC, Buckley CD, et al. Metabolic profiling predicts response to anti-tumor necrosis factor alpha therapy in patients with rheumatoid arthritis. Arthritis Rheum 2013;65:1448-56.

7. Priori R, Scrivo R, Brandt J, Valerio M, Casadei L, Valesini G, et al. Metabolomics in rheumatic diseases: the potential of an emerging methodology for improved patient diagnosis, prognosis, and treatment efficacy. Autoimmun Rev 2013; 12:1022-30.

8. Kang MJ, Park YJ, You S, Yoo SA, Choi S, Kim DH, et al. Urinary proteome profile predictive of disease activity in rheumatoid arthritis. J Proteome Res 2014;13:5206-17.

9. van der Heijde D. How to read radiographs according to the Sharp/van der Heijde method. J Rheumatol 2000;27:261-3.

10. Bruynesteyn K, van der Heijde D, Boers M, Saudan A, Peloso $\mathrm{P}$, Paulus $\mathrm{H}$, et al. Determination of the minimal clinically important difference in rheumatoid arthritis joint damage of the Sharp/van der Heijde and Larsen/Scott scoring methods by clinical experts and comparison with the smallest detectable difference. Arthritis Rheum 2002;46:913-20.

11. Dieterle F, Ross A, Schlotterbeck G, Senn H. Probabilistic quotient normalization as robust method to account for dilution of complex biological mixtures. Application in $1 \mathrm{H}$ NMR metabonomics. Anal Chem 2006;78:4281-90.

12. Trygg J, Wold S. Orthogonal projections to latent structures (O-PLS). J Chemom 2002;16:119-28.

13. Cloarec O, Dumas ME, Trygg J, Craig A, Barton RH, Lindon JC, et al. Evaluation of the orthogonal projection on latent structure model limitations caused by chemical shift variability and improved visualization of biomarker changes in $1 \mathrm{H}$ NMR spectroscopic metabonomic studies. Anal Chem 2005;77:517-26.

14. Bylesjö M, Rantalainen M, Cloarec O, Nicholson JK, Holmes E, Trygg J. OPLS discriminant analysis: combining the strengths of PLS-DA and SIMCA classification. J Chemom 2006;20:341-51.

15. Brand DD, Latham KA, Rosloniec EF. Collagen-induced arthritis. Nat Protoc 2007;2:1269-75.

16. Kim WU, Lee WK, Ryoo JW, Kim SH, Kim J, Youn J, et al. Suppression of collagen-induced arthritis by single administration of poly(lactic-co-glycolic acid) nanoparticles entrapping type II collagen: a novel treatment strategy for induction of oral tolerance. Arthritis Rheum 2002;46: 1109-20.

17. Kong JS, Yoo SA, Kim JW, Yang SP, Chae CB, Tarallo V, et al. Anti-neuropilin-1 peptide inhibition of synoviocyte survival, angiogenesis, and experimental arthritis. Arthritis Rheum 2010;62:179-90.

18. Wang Z, Klipfell E, Bennett BJ, Koeth R, Levison BS, Dugar $\mathrm{B}$, et al. Gut flora metabolism of phosphatidylcholine promotes cardiovascular disease. Nature 2011;472:57-63.

19. Mantovani A, Sica A, Sozzani S, Allavena P, Vecchi A, Locati $M$. The chemokine system in diverse forms of macrophage activation and polarization. Trends Immunol 2004;25:677-86.

20. Udalova IA, Mantovani A, Feldmann M. Macrophage heterogeneity in the context of rheumatoid arthritis. Nat Rev Rheumatol 2016;12:472-85.

21. Seldin MM, Meng Y, Qi H, Zhu W, Wang Z, Hazen SL, et al. Trimethylamine $\mathrm{N}$-Oxide promotes vascular inflammation through signaling of mitogen-activated protein kinase and nuclear factor-kappaB. J Am Heart Assoc 2016;5. pii: e002767.

22. Bain MA, Fornasini G, Evans AM. Trimethylamine: metabolic, pharmacokinetic and safety aspects. Curr Drug Metab 2005;6:227-40.

23. Zhang X, Zhang D, Jia H, Feng Q, Wang D, Liang D, et al. The oral and gut microbiomes are perturbed in rheumatoid arthritis and partly normalized after treatment. Nat Med 2015;21:895-905.

24. Scher JU, Sczesnak A, Longman RS, Segata N, Ubeda C, Bielski C, et al. Expansion of intestinal Prevotella copri correlates with enhanced susceptibility to arthritis. Elife 2013;2:e01202.

25. Koeth RA, Wang Z, Levison BS, Buffa JA, Org E, Sheehy BT, et al. Intestinal microbiota metabolism of L-carnitine, a nutrient in red meat, promotes atherosclerosis. Nat Med 2013;19:576-85.

26. Maier L, Pruteanu M, Kuhn M, Zeller G, Telzerow A, Anderson EE, et al. Extensive impact of non-antibiotic drugs on human gut bacteria. Nature 2018;555:623-8.

27. Vanholder R, Bammens B, de Loor H, Glorieux G, Meijers B, Schepers E, et al. Warning: the unfortunate end of p-cresol 
as a uraemic toxin. Nephrol Dial Transplant 2011;26: 1464-7.

28. Ligabue G, Damiano F, Cuoghi A, De Biasi S, Bellei E, Granito M, et al. p-Cresol and cardiovascular risk in kidney transplant recipients. Transplant Proc 2015;47:2121-5.

29. Yang XY, Zheng KD, Lin K, Zheng G, Zou H, Wang JM, et al. Energy metabolism disorder as a contributing factor of rheumatoid arthritis: a comparative proteomic and metabolomic study. PLoS One 2015;10:e0132695.

30. Biniecka M, Canavan M, McGarry T, Gao W, McCormick J, Cregan S, et al. Dysregulated bioenergetics: a key regulator of joint inflammation. Ann Rheum Dis 2016;75:2192-200.

31. Yang Z, Matteson EL, Goronzy JJ, Weyand CM. T-cell metabolism in autoimmune disease. Arthritis Res Ther 2015;
17:29.

32. Tak PP, Smeets TJ, Daha MR, Kluin PM, Meijers KA, Brand $\mathrm{R}$, et al. Analysis of the synovial cell infiltrate in early rheumatoid synovial tissue in relation to local disease activity. Arthritis Rheum 1997;40:217-25.

33. Wu GD, Chen J, Hoffmann C, Bittinger K, Chen YY, Keilbaugh SA, et al. Linking long-term dietary patterns with gut microbial enterotypes. Science 2011;334:105-8.

34. Cho CE, Taesuwan S, Malysheva OV, Bender E, Tulchinsky $\mathrm{NF}$, Yan J, et al. Trimethylamine-N-oxide (TMAO) response to animal source foods varies among healthy young men and is influenced by their gut microbiota composition: a randomized controlled trial. Mol Nutr Food Res 2017;61. DOI: $10.1002 / \mathrm{mnfr} .201600324$. 


\section{SUPPLEMENTARY DATA}

\section{${ }^{1}$ H NMR Spectroscopy of Urine Samples}

Urine samples were prepared for NMR analysis by mixing $440 \mu \mathrm{L}$ of urine with $220 \mu \mathrm{L}$ of phosphate buffer (pH=7.0, which included $0.1 \%$ sodium azide to prevent bacterial contamination and $1 \mathrm{mM}$ sodium 3-trimethylsilyl [2,2,3,3- $\left.{ }^{2} \mathrm{H}_{4}\right]$-1-propionate, TSP, in $\mathrm{D}_{2} \mathrm{O}$ ). The mixture was centrifuged to remove suspended particles. $550 \mu \mathrm{L}$ of the supernatant was transferred to an NMR tube. The ${ }^{1} \mathrm{H}$ NMR spectra of the urine samples were acquired using a Bruker 500 $\mathrm{MHz}$ spectrometer equipped with a cryogenic triple-resonance probe and an automatic sample changer (Bruker Biospin, Rheinstetten, Germany) operating at $500.13 \mathrm{MHz}{ }^{1} \mathrm{H}$ frequency and $300^{\circ} \mathrm{K}$. The "noesypresat" pulse sequence was used to suppress the water signal and to acquire the NMR spectrum. For each sample, 64 transients were collected into $32 \mathrm{~K}$ data points using a spectrum of $6,510.4 \mathrm{~Hz}$ with a relaxation delay of $2.0 \mathrm{~s}$ and a mixing time of $100 \mathrm{~ms}$. A line-broadening function of $0.3 \mathrm{~Hz}$ was applied to all spectra prior to the Fourier transformation. The phase and base line of the resultant NMR spectra were manually corrected. The chemical shift was calibrated to $\delta 0.00$ using the signal from the TSP with the phosphate buffer. 
Jung Hee Koh et al.

Supplementary Table 1. Sequences of the gene specific primers used for real-time PCR

\begin{tabular}{lll}
\hline \hline Gene & \multicolumn{1}{c}{ Forward } & \multicolumn{1}{c}{ Reverse } \\
\hline IL-6 & TTCCATCCAGTTGCCTTCTTG & AGGTCTGTTGGGAGTGGTATC \\
Arg1 & CTCCAAGCCAAAGTCCTTAGAG & AGGAGCTGTCATTAGGGACATC \\
Nos2 & GTTCTCAGCCCAACAATACAAGA & GTGGACGGGTCGATGTCAC \\
GAPDH & AACTTTGGCATTGTGGAAGG & GGATGCAGGGATGATGTTCT \\
\hline
\end{tabular}

PCR: polymerase chain reaction, IL: interleukin, Arg1: arginase 1, Nos2: nitric oxide synthase 2, GAPDH: glyceraldehyde 3-phosphate dehydrogenase.
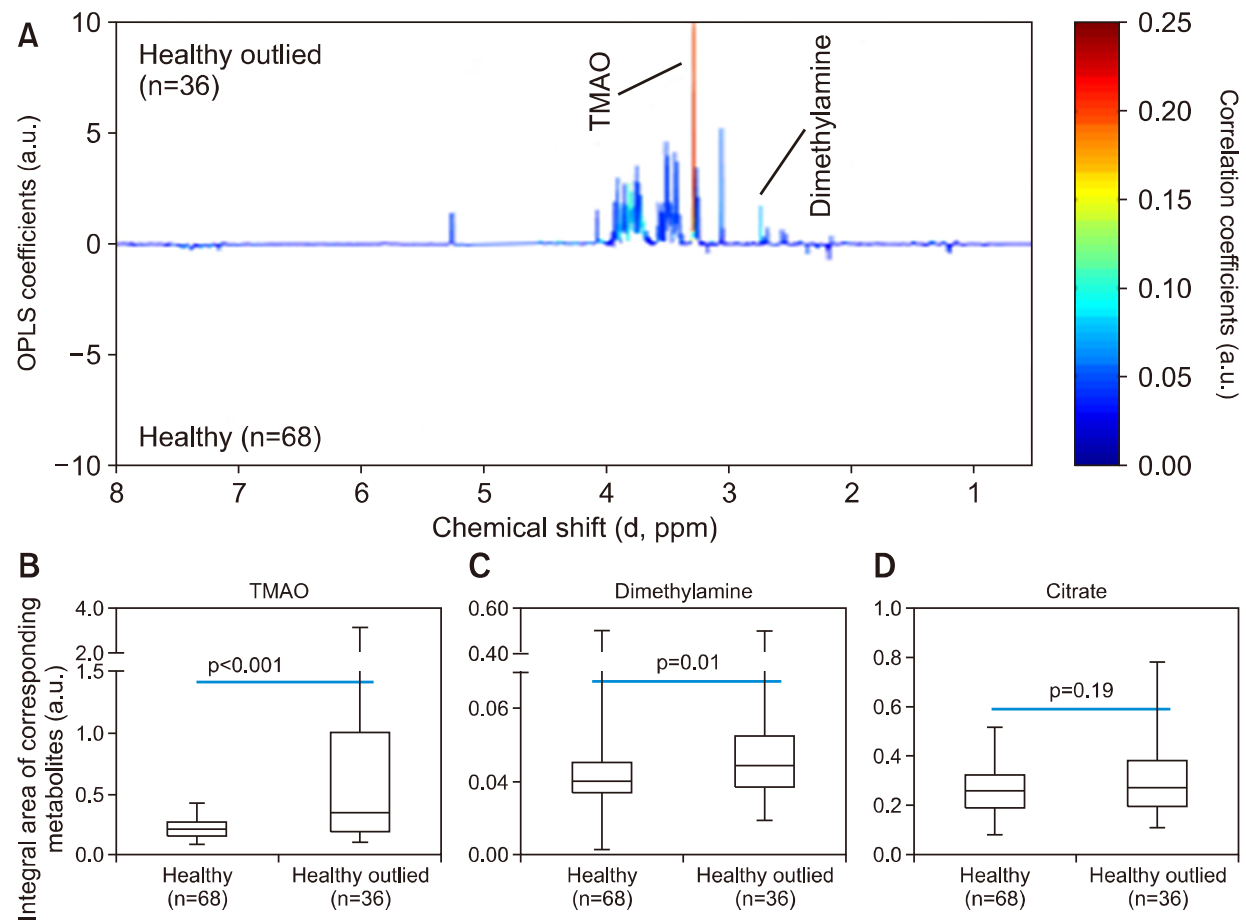

Supplementary Figure 1. Variations in the levels of urinary TMAO and dimethylamine between healthy volunteers who were outliers and those who were not in the multivariate statistical analysis from Figure 1. (A), the OPLS loading plot between the two classes; (B), statistical analysis of urinary TMAO; (C), statistical analysis of urinary dimethylamine; (D), statistical analysis of urinary citrate. TMAO: trimethylamine-N-oxide, OPLS: orthogonal projections to the latent structures, a.u.: arbitrary unit. 